\title{
Removal of Autogenous Fat Filling in Double Eyelid Operation by Artificial Intelligence (AI) Algorithm-Based Computerized Tomography (CT) Image Features
}

\author{
Tao Fang $\mathbb{D}^{1},{ }^{1}$ Wenwen Sui $\mathbb{D}^{2},{ }^{2}$ and Liang Guo $\mathbb{i}^{3}$ \\ ${ }^{1}$ Wuhan Wuchang Cascano Medical Beauty Clinic, Wuhan 430000, Hubei, China \\ ${ }^{2}$ Zhengzhou Erqi Xingchen Cosmetic Clinic, Zhengzhou 450000, Henan, China \\ ${ }^{3}$ Department of Plasticsurgery, Union Hospital, Tongji Medical College, Huazhong University of Science and Technology, \\ Wuhan 430022, Hubei, China \\ Correspondence should be addressed to Liang Guo; 3115101049@m.fafu.edu.cn
}

Received 23 November 2021; Revised 12 January 2022; Accepted 13 January 2022; Published 11 February 2022

Academic Editor: M Pallikonda Rajasekaran

Copyright (c) 2022 Tao Fang et al. This is an open access article distributed under the Creative Commons Attribution License, which permits unrestricted use, distribution, and reproduction in any medium, provided the original work is properly cited.

\begin{abstract}
The effect of autogenous fat filling was evaluated by computerized tomography (CT) image features based on the symmetric extended convolutional residual network image denoising algorithm in this research, and the pathological examination was conducted for the lesions of patients with complications. The examination provided more valid research basis for the clinical application of autogenous fat filling. 60 patients who received double eyelid operation were selected as the research objects, and the patients were randomly divided into the control group and the experimental group, where each group included 30 cases. The conventional double eyelid operation was adopted in treating the cases in the control group, while autogenous fat filling was adopted in the treatment of the cases in the experimental group. All patients in two groups were examined by CT images based on the symmetric extended convolutional residual network image denoising algorithm, and the therapeutic effects on patients in two groups and the evaluation of complications were compared. Next, the lesions of the complications of patients in the experimental group were examined pathologically. Besides, the efficacy and security of the pathological examination were assessed. The result showed that the values of peak signal-to-noise ratio (PSNR) and structural similarity (SSIM) $(\sigma$ (noise level) $=60: 24.78 \mathrm{~dB}$, $0.7022)$ in different noise images of the artificial intelligence algorithm adopted in the research were obviously higher than those obtained by the convolutional neural network $(\mathrm{CNN})$ and deep convolutional neural network $(\mathrm{DCNN})$ algorithms $(P<0.05)$. The therapeutic efficacy of patients in the experimental group was higher than that in the control group (68.67\% vs $60 \%)$. In addition, eyelid swelling scoring, double eyelid line width, and upper eyelid muscle strength of patients in the experimental group were all better than those of patients in the control group $(P<0.05)$. Besides, the incidence of complications $(10 \%)$ of patients in the experimental group was significantly lower than that $(30 \%)$ of patients in the control group. The pathological results of patients in the experimental group demonstrated that the lesion tissues might denature in future. As a result, the CT image processing of the algorithm adopted in this research could denoise effectively, and PSNR and SSIM values were high. In terms of the treatment by double eyelid operation, autogenous fat filling was effective, noninvasive, simple and resulted in low incidence of complications with a certain degree of security.
\end{abstract}

\section{Introduction}

To some extent, the sag or even sinking of upper eyelid is alleviated by double eyelid operation [1-3]. There are many types of methods of double eyelid operation, including mainly the incision method and the suture method. The incision method is commonly used in practice [4]. However, the incision method of double eyelid operation can beautify people's faces on the premise of success, but it may aggravate the sag of upper eyelid if the operation fails and even cause a series of complications [5]. Therefore, autogenous fat filling is proposed. 
Autogenous fat is viewed as the most natural and safest noninvasive filling material $[6,7]$ because of its little rejection reaction and perfect fusion with patients themselves. Research showed that autogenous fat filling is widely adopted in the treatment of the sag of upper eyelid with little trauma, no rejection [8], and high security. This method can be applied not only in the treatment of the sag of upper eyelid but also in the filling treatment of other parts of the face $[9,10]$. However, some research demonstrated that autogenous fat filling causes complications, such as localized hematoma, infection, and necrosis caused by poor fat survival at the filling parts [11], especially swollen eyes.

Swollen eyes are usually examined by the imaging features in clinical practice, such as magnetic resonance imaging (MRI) and computerized tomography (CT) [12]. MRI can show the boundary between lesions and normal tissues, and CT can present the cystic nodules of lesions with vague boundary $[13,14]$. Therefore, the adoption of MRI in most clinical treatments reflects the monotony of examination methods. To make the examination methods of swollen eyes more diverse, an intelligence algorithm is adopted to improve CT images and the accuracy of imaging in ocular examination. Research points out that the deep convolutional neural network (DCNN) has positive effects on the extraction and recognition of image features and is adopted in denoising images successfully [15]. DCNN is more learnable compared with the traditional denoising algorithm [16]. As a result, a symmetric extended convolutional residual network (SECRN) image denoising algorithm is proposed. Based on DCNN, symmetric extended technology is supplemented in SECRN. The results of the research demonstrate that the algorithm shows better denoising performance. It can not only remove the noises and artifacts in CT images but also retain the details of images [17].

To further explore the effects of the adoption of autogenous fat filling of double eyelid operation in treating the sag of upper eyelid, the imaging features were combined in the related analysis. The surgical effect of autogenous fat filling on patients was evaluated by CT images based on the symmetric extended convolutional residual network image denoising algorithm, and the lesions of patients with complications were examined. The results of these analyses provided more valid research bases for the clinical application of autogenous fat filling.

\section{Research Methods}

2.1. Research Objects. Sixty patients who received double eyelid operation in the hospital between March 2017 and March 2020 were chosen as research objects. All the patients were divided into two groups, including the control group and the experimental group. There were totally 30 cases in each group. The conventional double eyelid operation was adopted in the treatment of patients in the control group, and patients in the experimental group were treated by autogenous fat filling. Among 60 patients, there were 10 males and 50 females, ranging from 20 to 50 with the average of $(34.67 \pm 1.78)$. The therapeutic effects of two methods were evaluated by the comparison of the curative effects and complications of patients between two groups, and the properties of the lesions of patients with complications in the experimental group were detected. This study had been approved by the Ethics Committee of the hospital, and the patients and their families had known the research situation and signed the informed consent.

Inclusion standard: (a) patients without contradictions for the surgery in this research; (b) patients whose muscle strength of levator upper eyelid was higher than $8 \mathrm{~mm}$; (c) patients without receiving other surgical treatments in the past one month; (d) patients without infectious disease; (e) patients with high tolerance and compliance; (f) patients who were informed about and consented to the research.

Exclusion standard: (a) patients with trauma around eyes; (b) patients suffering from the dysfunction of the liver and kidney, and coagulation disorders; (c) patients with severe malignant tumors; (d) female patients in breastfeeding and pregnancy periods; (e) patients whose clinical data are incomplete.

2.2. Examination Methods. Examination apparatus was 16slice CT scanners, patient position was supine position, and scan position was on the scan baselines paralleling to orbital downlines. Scanning occurred on cross-sectional sides, coronal planes, and sagittal planes. Images in three groups were reconstructed. Scan parameter: the width of window was $350 \mathrm{H}$, window level was +30 , and the thickness was $2 \mathrm{~m}$. The details of ocular lesions were observed, such as location, size, form, and boundaries. All the above examinations were conducted by the same physician, and images were inspected by two experienced physicians.

\subsection{Symmetric Extended Convolutional Residual Network} Image Denoising Algorithm. The denoising of the symmetric extended convolutional residual network image denoising algorithm consists of three steps. (a) The features of the input noise images are extracted and learned by the symmetric convolutional network. (b) The features of the extracted images are reconstructed. (c) The images and noises are separated effectively by integrated residual learning and batch standardization, and the residual images are output (the size remains the same).

The denoising algorithm aims mainly at value additive white Gaussian noise, which is defined as follows:

$$
Y=X+N \text {. }
$$

Here, $Y$ refers to images containing noises, $X$ means noiseless images, $N$ stands for the noisy images distributed at $\left(0, \sigma^{2}\right)$, and $\sigma$ refers to the standard deviation of noises. The potential noisy-free images are predicted by the learning mapping function $f(Y)=X$, and the corresponding loss function can be expressed as follows:

$$
L=\frac{1}{n} \sum_{i=1}^{n}\left(\frac{1}{w \times h} \sum_{j=1}^{w} \sum_{k=1}^{h}\left\|f_{i}(j, k)-X_{i}(j, k)\right\|^{2}\right) .
$$

Here, $f$ refers to the denoised images, $X$ means the original noiseless images, $n$ stands for the number of training 
samples, and $w \times h$ refers to the width and height of samples. Later, the error mapping $R(Y) \approx N$ is trained according to residual learning, and mean square errors between the expected images and the predicted images are calculated by $X=Y-R(Y)$. Mean square errors can be expressed as follows:

$$
\ell\left(w, b, X_{i}, Y_{i}\right)=\frac{1}{2 N} \sum_{i=1}^{n}\left\|R_{w, b}\left(Y_{i}\right)-\left(Y_{i}-x_{i}\right)\right\|_{F}^{2} .
$$

Here, $w, b$ means learnable parameters, $\left\{X_{i}, Y_{i}\right\}_{i=1}^{N}$ refers to the $N$ noisy images and denoised images, $\ell\left(w, b, X_{i}, Y_{i}\right)$ stands for $N$ predicted sample noise images, and $\sum_{i=1}^{n}\left\|R_{w, b}\left(Y_{i}\right)-\left(Y_{i}-x_{i}\right)\right\|_{F}^{2}$ refers to the cost function. To figure out the minimum value of equation (3), $w$ and $b$ are optimized iteratively and updated by the random gradient descent algorithm [18], which are shown in

$$
\begin{aligned}
& w_{\mathrm{ij}}^{(l+1)}=w_{\mathrm{ij}}^{(l)}-\alpha \frac{\partial}{\partial w_{\mathrm{ij}}^{(l)}} \ell(w, b) . \\
& b_{i}^{(l+1)}=b_{i}^{(l)}-\alpha \frac{\partial}{\partial b_{i}^{(l)}} \ell(w, b) .
\end{aligned}
$$

In equations (4) and (5), $\ell\left(w, b, X_{i}, Y_{i}\right)$ refers to the target loss function, $\alpha$ means the learning rate, $l$ stands for the ordinal number of layers where the number is in, and $\partial$ represents the iterative coefficient. The random gradient descent algorithm improves network performance and training speed, but the network structure should not be ignored. Batch standardization [19] and residual learning [20] are widely used in the design of the structure of CNN, especially the former one, which is defined as follows:

$$
\bar{Z}^{(i)}=\gamma Z_{\text {norm }}^{i}+\beta .
$$

Here, $\gamma$ and $\beta$ refer to adjustable parameters, $\bar{Z}$ means the distribution of $Z_{\text {norm }}$ after the adjustments of $\gamma$ and $\beta$. $Z_{\text {norm }}$ can be shown in the equation as follows:

$$
Z_{\text {norm }}^{i}=\frac{Z^{(i)}-\mu}{\sqrt{\sigma^{2}+\xi}}
$$

Here, $\xi$ refers to small parameters (denominator must not be 0 ), $Z_{\text {norm }}$ means regularization, $Z$ stands for neutrons before activation, $\mu$ represents sample average, and $\sigma^{2}$ means sample square errors. As a result, sample average and square errors can be shown as follows:

$$
\begin{aligned}
\mu & =\frac{1}{m} \sum_{i} Z^{i}, \\
\sigma^{2} & =\frac{1}{m} \sum_{i}\left(Z^{i}-\mu\right)^{2} .
\end{aligned}
$$

The denoising effect of models is measured by peak signal-to-noise ratio (PSNR) and structural similarity (SSIM) in the research. The measurement is expressed by

$$
\text { PSNR }=10 \log _{10}\left(\frac{M}{\|\bar{I}-I\|_{2}^{2}}\right)
$$

Here, I stands for noiseless diffusion weighted images (DWI), $\bar{I}$ means denoised images, and $M$ represents the number of pixels in images.

$$
\operatorname{SSIM}(x, y)=\frac{\left(2 \alpha_{x} \alpha_{y}+e_{1}\right)\left(2 \beta_{x y}+e_{2}\right)}{\left(\alpha_{x}^{2}+\alpha_{y}^{2}+e_{1}\right)\left(\beta_{x}^{2}+\beta_{y}^{2}+e_{2}\right)}
$$

Here, $\alpha_{x}$ refers to the average of $x, \alpha_{y}$ means the average of $y, \beta_{x}^{2}$ stands for the square errors of $x, \beta_{y}^{2}$ means the square errors of $y$, and $\beta_{x y}$ refers to the covariance of $x$ and $y$.

\subsection{Therapeutic Methods}

2.4.1. Control Group. The conventional double eyelid operation was adopted in the treatment. Firstly, all patients received blood routine and palpebralis function examinations related to surgical effects before surgery, and the targeted surgical treatment plan was made by the comprehensive assessment of patients' clinical manifestations. Secondly, double eyelid lines were drawn on patients' upper eyelids. In surgery, patients laid on their backs, and local anesthesia was adopted. After that, the upper eyelids were disinfected regularly, and the skin and subcutaneous tissue were cut through along the double eyelid lines. Then, the orbicularis oculi at the anterior meibomian gland were cut off, and fat was removed. Next, the blepharon was sutured to the skin incision and adjusted appropriately according to the radian of double eyelids. After surgery, the incision was bound up under pressure and cooled by ice pack. Besides, patients were treated by antibiotics for consecutive five days to avoid complications.

2.4.2. Experimental Group. Double blepharoplasty was performed by autogenous fat filling. Preoperative examination methods were the same with that adopted in the control group, including blood routine, levator muscle function of upper eyelids, comprehensive evaluation of diseases, and stipulation of targeted plan. In surgery, local anesthesia or combined intravenous anesthesia and local swelling anesthesia at the donor area were adopted. Besides, the inner thigh was suctioned repeatedly by a liposuction needle to absorb sufficient fat particles, which were then rinsed and filtered by salt water and purified for the use of injection. Based on the size of fat particles, multichannel and multilayer injection was conducted. $1 \mathrm{ml}$ of fat particles was injected into fat around and in the rim of eyes through 30 to 50 channels. After surgery, the surgical area was iced to reduce swelling. Patients were prohibited to press hard the surgical area within seven days after surgery. Besides, an antibiotic therapy was offered to patients for five consecutive days to avoid complications. 


\subsection{Evaluation Indexes}

(a) The surgical effects of patients in two groups after surgery were divided into significantly effective (the shape was satisfactory, and the line was smooth), effective (the shape was slightly satisfactory, and the line was slightly smooth), and invalid (the shape was unsatisfactory, and the line was not smooth).

(b) Degree of eyelid swelling, width of double eyelid lines, muscle strength of upper eyelids, and degree of swelling of tissue around eyes were graded by the four-level scoring method [20] four months after surgery. Specific scoring criteria are shown in Table 1.

(c) The incidence of the complications from seven days to six months after the surgery of patients in the two groups was observed. The complications included infection, hematoma, skin necrosis, and ecchymosis. The calculation method was shown as follows:

The incidence of complication $=$ the number of patients with complications/the total number of cases $\times 100 \%$.

(d) The tissues of lesions of patients with complications in the control group were extracted and examined pathologically to evaluate their properties.

2.6. Statistical Methods. The data were processed by statistical product and service solutions (SPSS) 24.0 software. Enumeration data were expressed by (\%), and the comparison was tested by $\chi^{2}$. Measurement data were expressed by $(x \pm s)$, and the comparison was tested by $t . P<0.05$ indicated that the differences had statistical meaning.

\section{Results}

\subsection{Comparison of Denoising Performance}

3.1.1. Comparison of the Results of PSNR. Figure 1 shows the PSNR values of the algorithm adopted in the research, traditional CNN algorithm, and DCNN algorithm when $\sigma$ (noise intensity) $=30,40,50$, and 60. According to Figure 1, PSNR value of the algorithm adopted in the research was $27.11 \mathrm{~dB}$, PSNR value of the CNN algorithm was $25.81 \mathrm{~dB}$, and PSNR value of the DCNN algorithm was $26.45 \mathrm{~dB}$ when $\sigma=30$. When $\sigma=60$, PSNR value of the algorithm adopted in the research was $24.78 \mathrm{~dB}$, PSNR value of the CNN algorithm was $23.20 \mathrm{~dB}$, and PSNR value of the DCNN algorithm was $23.32 \mathrm{~dB}$. The analysis demonstrated that the PSNR values of the three algorithms all decreased as the noise level rose. However, PSNR values of the algorithm adopted in the research were all higher than those of CNN and DCNN algorithms $(P<0.05)$.

3.1.2. Comparison of the Results of SSIM. Figure 2 demonstrates the comparison of SSIM results of three algorithms when $\sigma=30,40,50$, and 60 . When $\sigma=30$, SSIM value of the algorithm adopted in the research was 0.8121 , SSIM value of the CNN algorithm was 0.7601 , and SSIM value of DCNN was 0.7609 . When $\sigma=60$, SSIM value of the algorithm adopted in the research was 0.7022 , SSIM value of the CNN algorithm was 0.6712 , and SSIM value of DCNN was 0.6821 . The analysis showed that the changing trend of three algorithms and the results of comparison were consistent with PSNR value. All the data indicated that the denoising performance of the algorithm adopted in the research was the best $(P<0.05)$.

\subsubsection{Comparison of Denoising Effect Diagrams. Figure 3} shows the comparison of denoising effect diagrams of CT images of three algorithms when $\sigma=30,40,50$, and 60. Figure 3 demonstrates that the denoising effect that adopted the algorithm in the research was obviously better than that that adopted other two algorithms, and the resolution ratio of the denoising effect diagram that adopted the algorithm in the research was higher than other two diagrams with higher definition.

3.2. Comparison of General Data. The general clinical data of patients in two groups were compared. The general data of 5 male patients $(50 \%)$ in the control group and 25 female patients $(50 \%)$ in the control group were selected. In the experimental group, the general data of 5 male patients (50\%) and 25 female patients (50\%) were selected. The average age of patients in the control group was $(31.72 \pm 1.69)$, and the average age of patients in the experimental group was $(33.02 \pm 1.29)$. The related analysis and comparison showed that the comparison of the distribution of the above general clinical data of patients in two groups had no statistical significance $(P<0.05)$, which indicated the feasibility of the comparison in this research.

3.3. Comparison of Surgical Effects. Table 2 shows the statistics of therapeutic effects of patients in two groups. The therapeutic efficiency of patients in the control group was $66.67 \%$ and that in the experimental group reached $86.67 \%$. Obviously, the therapeutic efficiency of patients in the experimental group was higher than that in the control group $(P<0.05)$. The double eyelid operation on a female patient was taken as the example, which is shown in Figure 4 . The operation was successfully performed. CT images also demonstrated that there were no obvious nodules in eyelid areas.

3.4. Comparison of Clinical Indexes. Table 3 shows the scoring results of eyelid swelling scoring, width of double eyelids, and muscle strength of upper eyelids four months after treatment. The results demonstrated that the average of the scores of eyelid swelling scoring, width of double eyelids, and muscle strength of upper eyelids of patients in the experimental group were all better than those of patients in the control group $(P<0.05)$.

3.5. Incidence of Complications. Table 4 shows the incidence of infection, pruritus, hematoma, skin necrosis, and ecchymosis. According to Table 4, hematoma was the 
TABLE 1: Four-level scoring method of swelling of tissues around eyes.

\begin{tabular}{lccc}
\hline Levels & Degree of swelling & Changes of skin & Score \\
\hline Level 1 & No swelling & No change & 0 \\
Level 2 & Slight swelling & No cyanosis & 1 \\
Level 3 & Moderate swelling & Slight cyanosis and texture appeared & 2 \\
Level 4 & Severe swelling & Lots of cyanoses, skin became cold, and texture disappeared & 3 \\
\hline
\end{tabular}

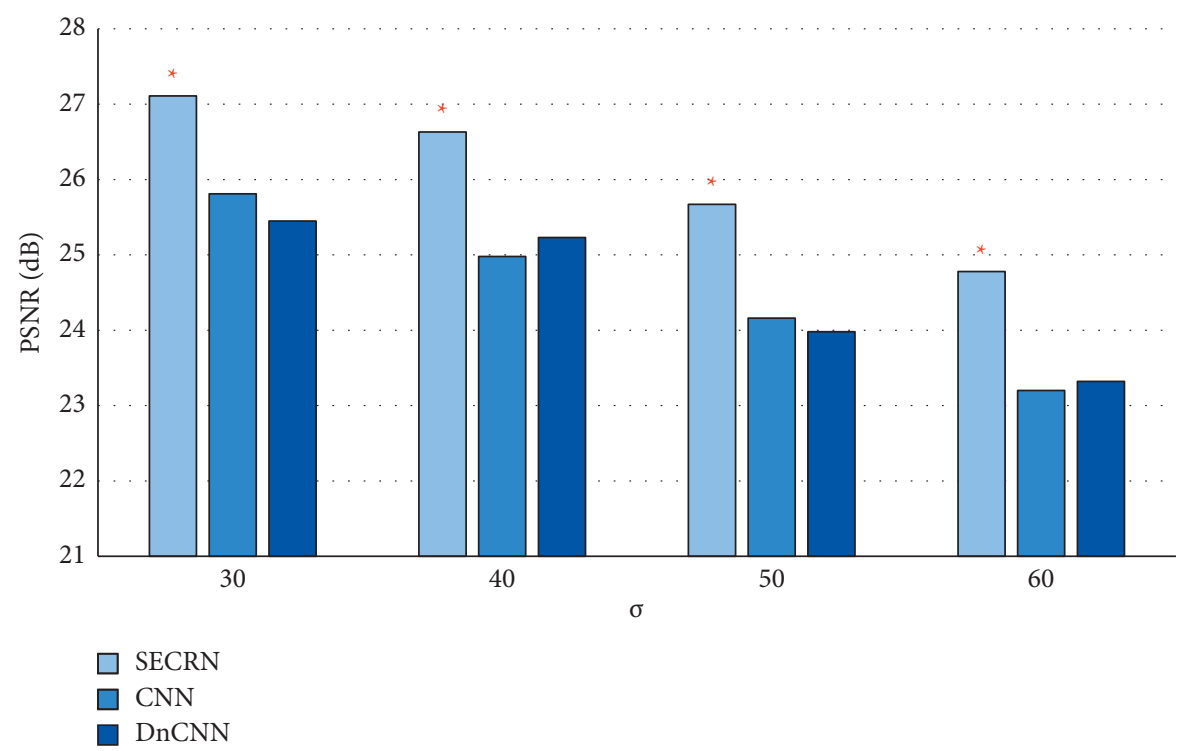

FIgURE 1: Comparison of PSNR values of three algorithms. *PSNR of SECRN algorithm with that of CNN and DCNN showed obvious differences $(P<0.05)$.

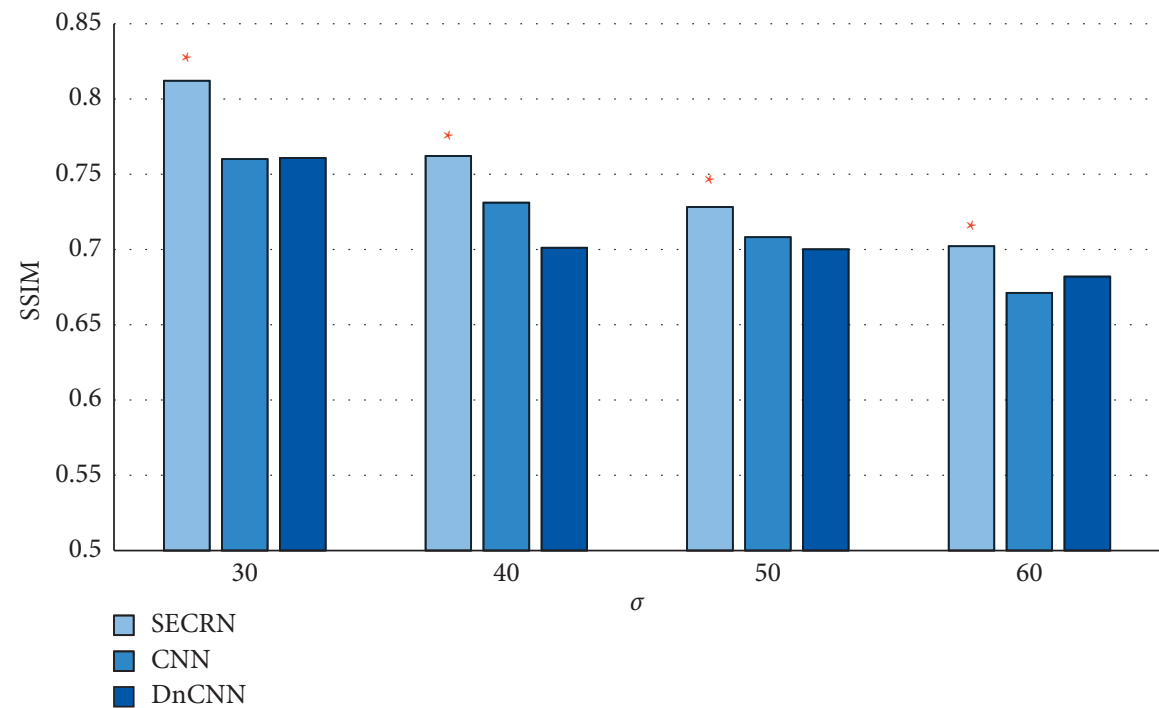

FIgURE 2: Comparison of SSIM results. $*$ SSIM of SECRN algorithm was obviously different from those of CNN and DCNN $(P<0.05)$.

commonest disease among the complications of patients in the experimental group (6.67\%), and infection was the commonest symptom among the complications of patients in the control group (23.33\%). In contrast, there was no skin necrosis among patients in the experimental group. Besides, the total incidence of all complications in the control group (73.33\%) was significantly higher than that in the experimental group (6\%). In terms of the number of patients with complications, there were 9 cases in the control group and 3 cases in the experimental group. The related calculation and 


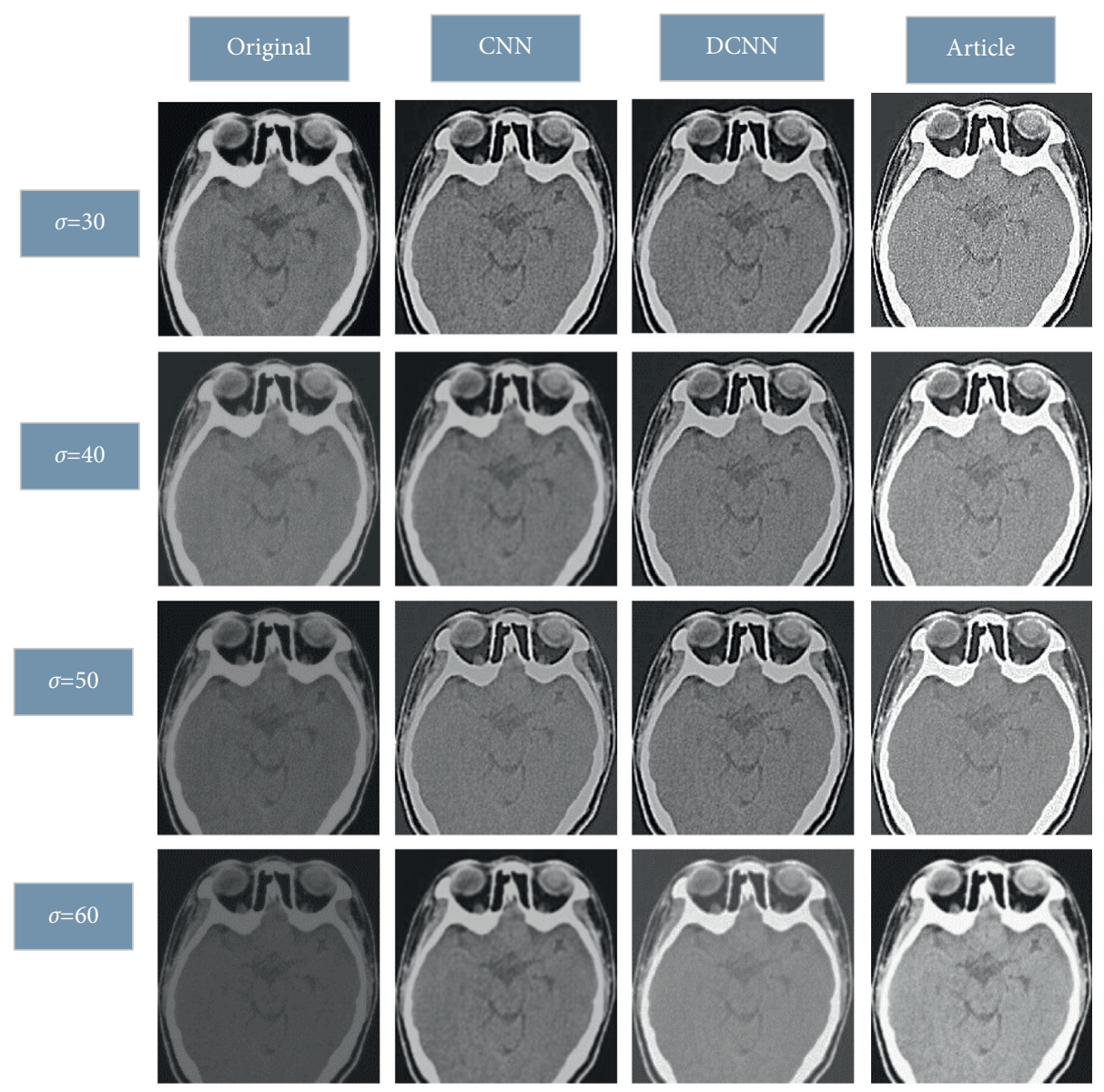

FIGURE 3: Denoising effect diagrams.

TABLE 2: Statistics of therapeutic effects.

\begin{tabular}{lccc}
\hline Therapeutic effect & Control group $(n=30$ cases $)$ & Experimental group $(n=30$ cases $)$ & Total number \\
\hline Significantly effective & 12 & 18 & 28 \\
Slightly effective & 8 & 8 & 16 \\
Invalid & 10 & 4 & 14 \\
Efficiency & $66.67 \% *$ & $86.67 \% *$ & $73.33 \%$ \\
\hline
\end{tabular}

* The comparison showed statistical differences $(P<0.05)$.
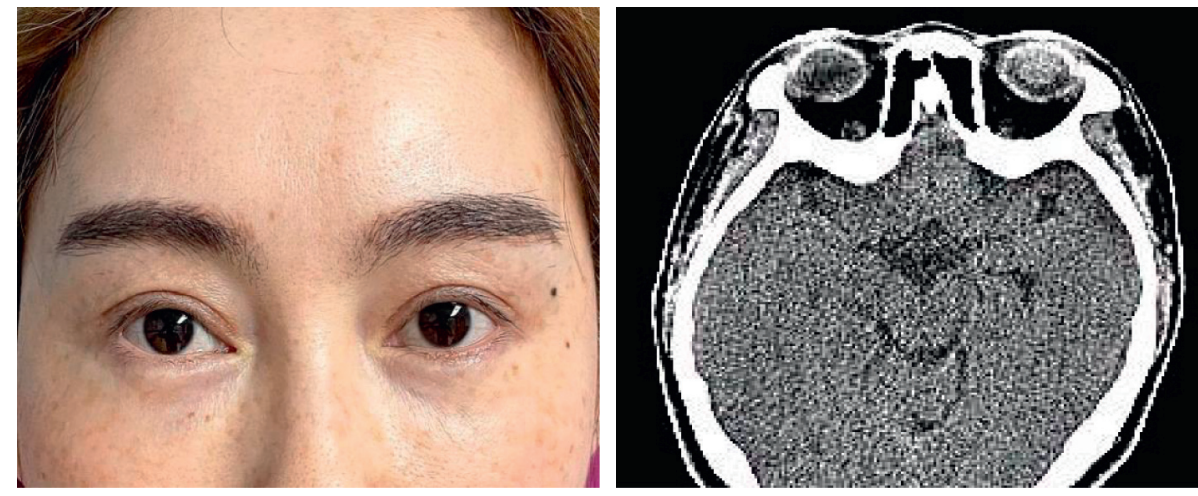

FIGURE 4: Therapeutic effects of a patient in the experimental group (female, 35 years old, and purpose of surgery: double-fold eyelids). 
TABLE 3: Statistics of results of clinical indexes.

\begin{tabular}{|c|c|c|c|}
\hline \multirow{2}{*}{ Indexes } & \multicolumn{2}{|c|}{ Evaluation results } & \multirow{2}{*}{$P$} \\
\hline & Experimental group ( $n=30$ cases) & Control group ( $n=30$ cases) & \\
\hline Eyelid swelling (score) & $0.10 \pm 0.01$ & $0.23 \pm 0.04$ & $0.001 *$ \\
\hline Width of double eyelids (mm) & $4.48 \pm 0.59$ & $3.45 \pm 0.46$ & $0.002 *$ \\
\hline Muscle strength of upper eyelids (mm) & $14.08 \pm 2.07$ & $10.98 \pm 1.57$ & $0.001 *$ \\
\hline
\end{tabular}

*The comparison showed statistical differences $(P<0.05)$.

TABLE 4: Statistics of the incidence of all complications.

\begin{tabular}{lcccc}
\hline \multirow{2}{*}{$\begin{array}{l}\text { Complications } \\
\end{array}$} & Experimental group $(n=30$ cases $)$ & Proportion $(\%)$ & Control group $(n=30$ cases) & Proportion $(\%)$ \\
\hline Infection & 1 & 3.33 & 7 & 23.33 \\
Pruritus & 1 & 3.33 & 5 & 13.33 \\
Hematoma & 3 & 6.67 & 3 & 16.67 \\
Skin necrosis & 0 & 0 & 5 & 10 \\
Ecchymosis & 1 & 3.33 & 22 & 73.67 \\
Total & 6 & $20 *$ & $53 *$ \\
\hline
\end{tabular}

*The comparison showed statistical differences $(P<0.05)$.

analysis indicated that the incidence of complications of patients in the experimental group (10\%) was obviously lower than that of patients in the control group $(30 \%)$ $(P<0.05)$.

3.6. Pathological Results of Patients with Complications in the Experimental Group. Figure 5 shows the manifestations of normal eyelids and eyelids with hematoma in CT images. Compared with normal CT images of eyes, high signal shadows appeared in the soft tissues in the left eyes of patients with complications, and the density inside the shadows was uneven (marked by the yellow arrow). The tissues in the lesions of this patient were extracted (as shown in Figure 6(a)) and examined pathologically (as shown in Figure 6(b)), and there were hemorrhage and denaturation in local areas of the extracted tissues. The discovery implied that the complications appearing after autogenous fat filling may cause the denaturation of the tissues, which was risky.

\section{Discussion}

With the growing widespread clinical application of autogenous fat filling in cosmetology, its security and efficacy drew people's attention widely [21]. The research also focused on the security and efficacy of this technology, and the adoption in double eyelid operation. The security and efficacy of autogenous fat filling were examined by CT images. To improve the effectiveness of examination by CT images, an AI algorithm was adopted to denoise CT images. The algorithm used in denoising processing was the symmetric extended convolutional residual network image denoising algorithm, which was compared with CNN and DCNN algorithms. The results showed that SPNR and SSIM values of the algorithm in the research in different noise images ( $\sigma=60: 24.78 \mathrm{~dB}, 0.7022)$ were obviously higher than those of $\mathrm{CNN}$ and DCNN algorithms $(P<0.05)$. Besides, it was proposed that the denoising methods of CNN and DCNN algorithms should resort to external information during a complex process over a long period of time, and the denoising effect on high-noise images was not significant enough [22]. In contrast, the symmetric extended convolutional residual network image denoising algorithm could solve the above problems, and the denoising effect of this algorithm was better according to related research results, which was consistent with the outcome of the research conducted by Yao et al. [23].

Autogenous fat filling and traditional double eyelid operation were compared in this research. The research proposed that the tissue activity of autologous fat was high, and postoperative cosmetic effect was satisfactory. Autogenous fat filling made skin become elastic, and lines look plump again $[24,25]$. Also, the research results showed that the therapeutic efficiency of patients in the experimental group was higher than that in the control group $(68.67 \%$ vs $60 \%$ ), and eyelid swelling scoring, width of double eyelids, and muscle strength of upper eyelids of patients in the experimental group $((0.10 \pm 0.01),(4.48 \pm 0.59) \mathrm{mm}$, and $(14.08 \pm 2.07) \mathrm{mm})$ were all better than those of patients in the control group $((0.23 \pm 0.04),(3.45 \pm 0.46) \mathrm{mm}$, and $(10.98 \pm 1.57) \mathrm{mm})(P<0.05)$. This result was consistent with the research results of Shue et al. [26]. According to the research, the commonest complication among patients in the experimental group was hematoma (6.67\%), and infection was the commonest one in the control group $(23.33 \%)$, which was related to the surgical incision. Waltz and Zuckerbraun [27] proposed that the size of surgical incision and postoperative nursing was closely related to incision infection. Injection filling was the main method adopted in the surgeries of patients in the experimental group, so hematoma was the commonest adverse reaction, as Toyoda et al. [28] found out. However, the research also pointed out that the incidence of adverse reactions of autogenous fat filling was low. This viewpoint was a supporting 


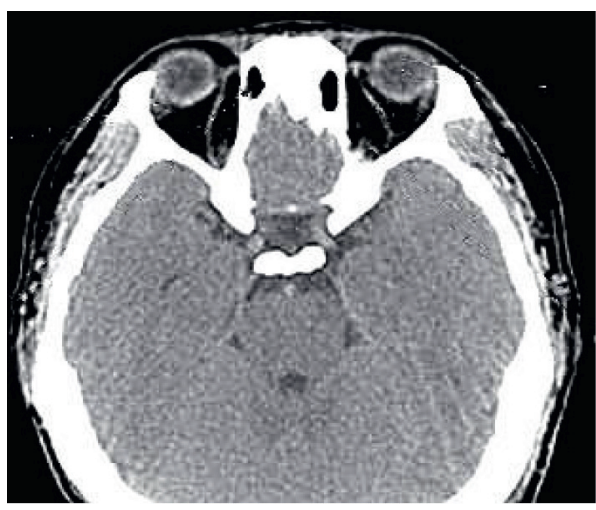

(a)

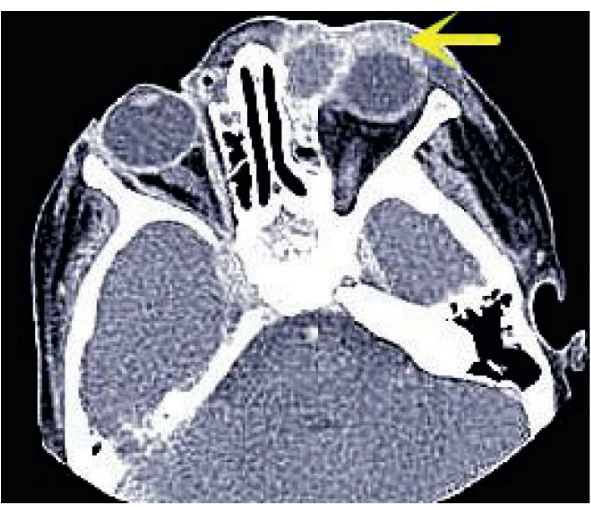

(b)

FIgURE 5: CT examination results. (a) Female patients with normal eyelids at 33. (b) Female patients with swollen eyelids at 36.

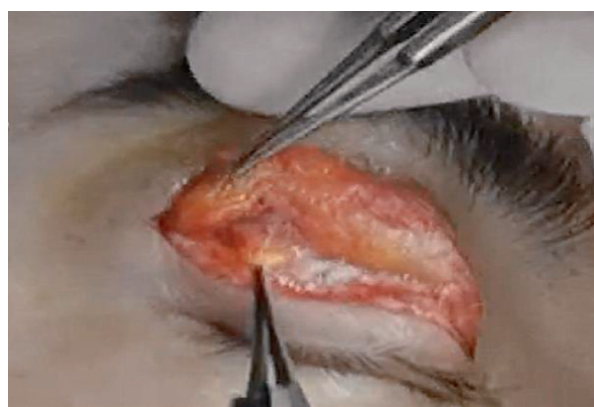

(a)

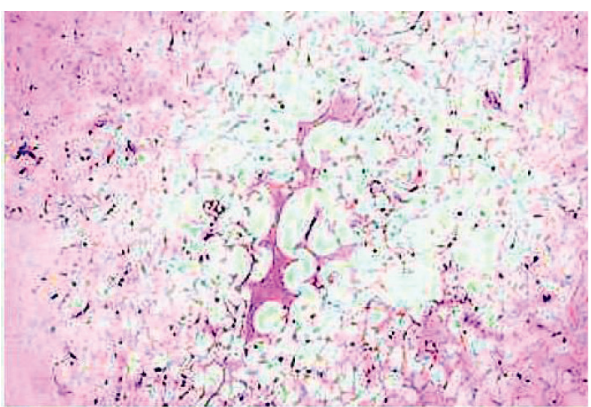

(b)

FIgURE 6: Results of lesion extraction (a) and pathological examination (b).

theoretical basis for the results of this research, which demonstrated that the incidence of complications of patients in the experimental group (10\%) was obviously lower than that of patients in the control group $(30 \%)(P<0.05)$. Later on, histopathologic biopsy was performed on patients with complications to further know about the security of the treatment by autogenous fat filling. Eventually, the results showed that lesion tissues might denature. However, denaturation seldom occurred based on the analysis of the above research results, and considerable research indicated that the incidence of the adverse reaction of denaturation was low, so the surgical treatment by autogenous fat filling was secure [29-31].

\section{Conclusion}

The therapeutic effects of autogenous fat filling were evaluated by CT images based on the symmetric extended convolutional residual network image denoising algorithm, and the lesions of patients with complications were examined pathologically. The results are shown as follows: (a) The algorithm in the research had more positive denoising effects on CT image processing than PSNR and SSIM, the noise levels of which were higher than the algorithm in the research. (b) The therapeutic efficiency of autogenous fat filling in double eyelid operation was high, the incidence of complications was low, and the adoption in surgical treatment was safe. Nevertheless, the factors that cause complications were not analyzed in the research, such as the relationship between infection and incision, and between skin necrosis and tissue activity. Further explorations would be made in future research. In general, autogenous fat filling had positive effects on double eyelid operation without severe complications. In addition, it could be adopted safely and exerted obvious effects in surgical treatment. Besides, it caused little trauma, and the operating procedure was simple with high value of clinical application.

\section{Data Availability}

The data used to support the findings of this study are available from the corresponding author upon request.

\section{Conflicts of Interest}

The authors declare no conflicts of interest.

\section{References}

[1] T. Mau and R. Yung, "Adipose tissue inflammation in aging," Experimental Gerontology, vol. 105, pp. 27-31, 2018 May.

[2] P. Xu, H. Huang, S. Zhang, X. Yin, Q. Zhang, and Y. Du, "A comprehensive approach to upper eyelid rejuvenation surgery," Aesthetic Plastic Surgery, vol. 45, no. 3, pp. 1047-1055, 2021 Jun. 
[3] K. K. Kim, W.-S. Kim, S. K. Oh, and H. S. Kim, "High double eyelid fold correction using wide dual-plane dissection," Annals of Plastic Surgery, vol. 78, no. 4, pp. 365-370, 2017 Apr.

[4] W. P. D. Chen, "Primary double eyelid surgery-trapezoidal debulking of upper eyelid tissues," Plastic and Reconstructive Surgery - Global Open, vol. 6, no. 5, Article ID e1780, 2018 May 15.

[5] C. Yang, X. Guo, L. Du et al., "A modified procedure for single-eyelid asian females with lacrimal gland prolapse: lacrimal gland reposition combined with fat transposition in double-eyelid operation," Aesthetic Plastic Surgery, vol. 45, no. 4, pp. 1611-1619, 2021 Aug.

[6] P. Bui and C. Lepage, "Apport de la volumétrie au rajeunissement facial. Partie 1: greffe adipocytaire," Annales de Chirurgie Plastique Esthétique, vol. 62, no. 5, pp. 532-549, 2017 Oct.

[7] F. Simonacci, N. Bertozzi, M. P. Grieco, E. Grignaffini, and E. Raposio, "Procedure, applications, and outcomes of autologous fat grafting," Annals of Medicine and Surgery, vol. 20, pp. 49-60, 2017 Jun 27.

[8] H. Chen, Q. Zhang, Q. Qiu, and Z. Yang, "Autologous fat graft for the treatment of sighted posttraumatic enophthalmos and sunken upper eyelid," Ophthalmic Plastic and Reconstructive Surgery, vol. 34, no. 4, pp. 381-386, 2018 Jul/Aug.

[9] W. Lee, S.-B. Kwon, S.-K. Oh, and E.-J. Yang, "Correction of sunken upper eyelid with orbital fat transposition flap and dermofat graft," Journal of Plastic, Reconstructive \& Aesthetic Surgery, vol. 70, no. 12, pp. 1768-1775, 2017 Dec.

[10] H. Chatel, B. Hersant, R. Bosc, S. La Padula, and J. P. Meningaud, "Midface rejuvenation surgery combining preperiosteal midcheek lift, lower blepharoplasty with orbital fat preservation and autologous fat grafting," Journal of Stomatology, Oral and Maxillofacial Surgery, vol. 118, no. 5, pp. 283-288, 2017 Oct.

[11] D. B. Hom and J. Harmon, "Investigating methods to prevent blindness from facial fat injections," JAMA Facial Plastic Surgery, vol. 20, no. 6, pp. 451-452, 2018 Dec 1.

[12] Y. Ito, I. Yamazaki, Y. Kikuchi et al., "Imaging characteristics of the postoperative globe: a pictorial essay," Japanese Journal of Radiology, vol. 34, no. 12, pp. 779-785, 2016 Dec.

[13] F. Heran Dreyfus, O. Galatoire, P. Koskas, F. Lafitte, E. Nau, and O. Bergès, "Pathologie vasculaire orbito-palpébrale," Journal Français d'Ophtalmologie, vol. 39, no. 9, pp. 804-813, 2016 Nov.

[14] N. Nishio, Y. Fujimoto, M. Hiramatsu et al., "Computed tomographic assessment of autologous fat injection augmentation for vocal fold paralysis," Laryngoscope Investigative Otolaryngology, vol. 2, no. 6, pp. 459-465, 2017 Nov 22.

[15] M. Hu, Y. Zhong, S. Xie, H. Lv, and Z. Lv, "Fuzzy system based medical image processing for brain disease prediction," Frontiers in Neuroscience, vol. 15, Article ID 714318, $2021 \mathrm{Jul}$ 30.

[16] Li Yi, J. L. Zhao, Z. H. Lv, and J. Li, "Medical image fusion method by deep learning," International Journal of Cognitive Computing in Engineering, vol. 15, no. 5, pp. 443-452, 2021.

[17] Z. Feng, A. Cai, Y. Wang, L. Li, L. Tong, and B. Yan, "Dual residual convolutional neural network (DRCNN) for lowdose CT imaging," Journal of X-Ray Science and Technology, vol. 29, no. 1, pp. 91-109, 2021.

[18] L. Wang, Y. Yang, R. Min, and S. Chakradhar, "Accelerating deep neural network training with inconsistent stochastic gradient descent," Neural Networks, vol. 93, pp. 219-229, 2017 Sep.
[19] D. S. Bassett and O. Sporns, "Network neuroscience," Nature Neuroscience, vol. 20, no. 3, pp. 353-364, 2017 Feb 23.

[20] K. Yang, W. Zhang, X. Chen et al., "[Establishment and preliminary application of tissue response grading system following neoadjuvant chemotherapy]," Zhonghua Wei Chang Wai Ke Za Zhi, vol. 21, no. 9, pp. 1032-1038, 2018 Sep 25.

[21] S. Zhang, K. Ding, X. Bai, Z. Cao, and X. Liang, "Orbital septum fat flap transfer for upper eyelid depression," Journal of Craniofacial Surgery, vol. 33, no. 1, pp. e52-e54, 2021 Jul 20.

[22] K. Zhang, W. Zuo, Y. Chen, D. Meng, and L. Zhang, "Beyond a Gaussian denoiser: residual learning of deep CNN for image denoising," IEEE Transactions on Image Processing, vol. 26, no. 7, pp. 3142-3155, 2017 Jul.

[23] N. M. Yao, Q. P. Guo, F. C. Qiao, H. Chen, and H. A. Wang, "Robust facial expression recognition with generative adversarial networks[J]," Zidonghua Xuebao/Acta Automatica Sinica, vol. 44, no. 5, pp. 865-877, 2018.

[24] K. Arnautovic and M. Kovacevic, "CSF-related complications after intradural spinal tumor surgery: utility of an autologous fat graft," Medical Archives, vol. 70, no. 6, pp. 460-465, 2016 Dec.

[25] M. Aron, T. G. Landry, and M. Bance, "Auditory effects of autologous fat graft for TORP stabilization in the middle ear: a cadaveric study," Journal of Otolaryngology - Head \& Neck Surgery, vol. 47, no. 1, p. 17, 2018 Feb 17.

[26] S. Shue, D. E. Kurlander, and B. Guyuron, "Fat injection: a systematic review of injection volumes by facial subunit," Aesthetic Plastic Surgery, vol. 42, no. 5, pp. 1261-1270, 2018 Oct.

[27] P. K. Waltz and B. S. Zuckerbraun, "Surgical site infections and associated operative characteristics," Surgical Infections, vol. 18, no. 4, pp. 447-450, 2017 May/Jun.

[28] Y. Toyoda, K.-B. Celie, J. T. Xu et al., "A 3-dimensional biomimetic platform to interrogate the safety of autologous fat transfer in the setting of breast cancer," Annals of Plastic Surgery, vol. 80, no. 4, pp. S223-S228, 2018 Apr.

[29] C.-Y. Chiu, Y.-C. Shen, Q.-F. Zhao, F.-L. Hong, and J.-H. Xu, "Treatment of tear trough deformity: fat repositioning versus autologous fat grafting," Aesthetic Plastic Surgery, vol. 41, no. 1, pp. 73-80, 2017 Feb.

[30] X. Wang, M. Wu, X. Zhou, H. Liu, Y. Zhang, and H. Wang, "Autologous fat used for facial filling can lead to massive cerebral infarction through middle cerebral artery or facial intracranial branches," Journal of Craniofacial Surgery, vol. 29, no. 5, pp. 1341-1343, 2018 Jul.

[31] S. Liu, X. Chen, Y. Su et al., "Association of autologous fat injection in facial artery with ophthalmological complications," JAMA Facial Plastic Surgery, vol. 20, no. 6, pp. 445-451, 2018 Dec 1. 